\title{
HOLONOMY LIE ALGEBRAS AND THE LCS FORMULA FOR SUBARRANGEMENTS OF $A_{n}$
}

\author{
PAULO LIMA-FILHO AND HAL SCHENCK ${ }^{1}$
}

\begin{abstract}
If $X$ is the complement of a hypersurface in $\mathbb{P}^{n}$, then Kohno showed in 9 that the nilpotent completion of $\pi_{1}(X)$ is isomorphic to the nilpotent completion of the holonomy Lie algebra of $X$. When $X$ is the complement of a hyperplane arrangement $\mathcal{A}$, the ranks $\phi_{k}$ of the lower central series quotients of $\pi_{1}(X)$ are known in only two very special cases: if $X$ is hypersolvable (in which case the quadratic closure of the cohomology ring is Koszul), or if the holonomy Lie algebra decomposes in degree 3 as a direct product of local components. In this paper, we use the holonomy Lie algebra to obtain a formula for $\phi_{k}$ when $\mathcal{A}$ is a subarrangement of $A_{n}$. This extends Kohno's result 10] for braid arrangements, and provides the first instance of an LCS formula for arrangements which are not decomposable or hypersolvable.
\end{abstract}

\section{INTRODUCTION}

Let $X$ be the complement of an arrangement of projective hypersurfaces. Associated to the fundamental group $\pi_{1}(X)$ of $X$ is the lower central series: a chain of normal subgroups

$$
\pi_{1}(X)=G_{1} \geq G_{2} \geq G_{3} \geq \cdots,
$$

defined inductively by $G_{k}=\left[G_{k-1}, G_{1}\right]$. The graded vector space $\left(\oplus G_{i} / G_{i+1}\right) \otimes \mathbb{Q}$ becomes a graded Lie algebra over $\mathbb{Q}$ with bracket given by $[x, y]=x y x^{-1} y^{-1}$. In 9], Kohno combined results of Deligne [4] and Morgan [1] on the bigrading on the mixed Hodge structure of $H^{1}(X, \mathbb{Q})$ with results of Sullivan [21] on the minimal model to show that $\left(\oplus G_{i} / G_{i+1}\right) \otimes \mathbb{Q}$ is isomorphic to the nilpotent completion of the holonomy Lie algebra of $X$.

If $X$ is the complement of an arrangement of hyperplanes $\mathcal{A}$, the holonomy Lie algebra $\mathfrak{h}_{X}$ is a purely combinatorial object, defined in terms of the rank two flats in the intersection lattice $\mathcal{L}(\mathcal{A})$. Elements of rank $i$ in $\mathcal{L}(\mathcal{A})$ correspond to maximal sets of hyperplanes $\left\{H_{1}, \ldots, H_{k}\right\}$ intersecting in codimension exactly $i$. Slicing an arrangement $\mathcal{A}$ with a generic plane yields an arrangement $\mathcal{A}^{\prime} \subseteq \mathbb{P}^{2}$ with $\mathcal{L}(\mathcal{A}) \simeq \mathcal{L}\left(\mathcal{A}^{\prime}\right)$ in rank $\leq 2$. In a similar vein, a theorem of Hamm-Lê [7] shows that the fundamental group of $X$ is unaffected by the process of slicing down generically to an arrangement in $\mathbb{P}^{2}$, which shows that the algebra $\left(\oplus G_{i} / G_{i+1}\right) \otimes \mathbb{Q}$ depends only on a generic planar restriction of $X$. Comparing these two reductions suggests the possibility of a result like that proved by Kohno. An immediate consequence of the isomorphism $\mathfrak{h}_{X} \simeq\left(\oplus G_{i} / G_{i+1}\right) \otimes \mathbb{Q}$ is that $\phi_{k}$ is the dimension of the $k^{t h}$ graded piece of $\mathfrak{h}_{X}$, where $\mathfrak{h}_{X}$ is graded by bracket depth.

Date: October 5, 2008.

2000 Mathematics Subject Classification. Primary 52C35; 20F14, 20F40,

Key words and phrases. holonomy Lie algebra, lower central series, hyperplane arrangement.

${ }^{1}$ Partially supported by NSF DMS 07-07667, NSA MDA 904-03-1-0006. 
In [10, Kohno used this framework to prove a beautiful formula for the LCS ranks of the braid arrangement $A_{n}$ :

$$
\prod_{k=1}^{\infty}\left(1-t^{k}\right)^{\phi_{k}}=P(X,-t)=\prod_{i=1}^{n-1}(1-i t)
$$

where $P(X, t)=\sum b_{i} t^{i}$ is the Poincaré polynomial of the complement of $X$ in $\mathbb{C}^{n}$.

Definition 1.1. Let $G$ be a (simple) graph on $\ell$ vertices, with edge-set $\mathrm{E}$, and let $\mathcal{A}_{G}=\left\{z_{i}-z_{j}=0 \mid(i, j) \in \mathrm{E}\right\}$ be the corresponding arrangement in $\mathbb{C}^{\ell} ; \mathcal{A}_{G}$ is called a graphic arrangement. Write $U_{G}(t)$ for $\prod_{k=1}^{\infty}\left(1-t^{k}\right)^{\phi_{k}\left(\pi_{1}\left(\mathbb{C}^{\ell} \backslash \mathcal{A}_{G}\right)\right)}$.

The braid arrangement $A_{n}$ is the arrangement associated to the graph $K_{n}$. Our main result is an extension (conjectured in [16]) of Kohno's theorem:

Theorem 1.2 (Graphic LCS formula). If $G$ is a graph with $\ell$ vertices, and $\kappa_{s}$ denotes the number of complete subgraphs of $G$ on $s+1$ vertices, then:

$$
U_{G}(t)=\prod_{j=1}^{\ell-1}(1-j t)^{\sum_{s=j}^{\ell-1}(-1)^{s-j}\left(\begin{array}{l}
s \\
j
\end{array}\right) \kappa_{s}}
$$

Example 1.3. Suppose $G$ consists of the 1-skeleton of the Egyptian pyramid and the one skeleton of a tetrahedron, sharing a single triangle, as below:

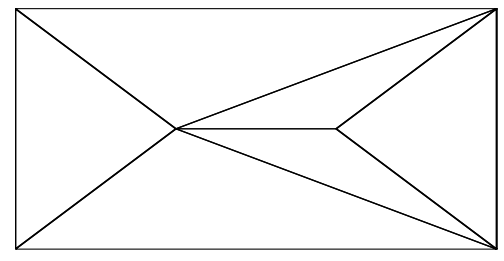

For this graph, $U_{G}(t)=(1-2 t)^{4}(1-3 t)$. The proof of $(1.2)$ uses a Mayer-Vietoris argument: a graph is determined by the data of the maximal complete subgraphs, together with gluing data. For example, if $G^{\prime}$ denotes the 1-skeleton of the Egyptian pyramid, then four triangles are glued along four edges, and Corollary 2.5 shows that $U_{G^{\prime}}(t)=((1-t)(1-2 t))^{4} /(1-t)^{4}=(1-2 t)^{4}$. For the $K_{4}$ subgraph, $U_{K_{4}}(t)=$ $(1-t)(1-2 t)(1-3 t)$. The graphs $G^{\prime}$ and $K_{4}$ are glued along a common triangle, hence

$$
U_{G}(t)=\frac{(1-2 t)^{4} \cdot(1-t)(1-2 t)(1-3 t)}{(1-t)(1-2 t)} .
$$

1.4. Other LCS formulas. To place (1.2) in context, we note that Kohno's formula has previously been extended in two different directions. In [6], Falk and Randell give a generalization to fiber-type arrangements, i.e. those whose complement can be factored as a chain of linear fibrations with fiber $F_{i} \simeq \mathbb{C}-\left\{p_{1}, \ldots, p_{i_{k}}\right\}$. As shown by Terao in 22], this is equivalent to a lattice-theoretic condition known as supersolvability. Supersolvability implies that $H^{*}(X)$ admits a quadratic Gröbner basis, which implies that $H^{*}(X)$ is Koszul; in [18, Shelton and Yuzvinsky interpret the LCS formula (1.1) in terms of Koszul duality. The requirement that $H^{*}(X)$ be Koszul can be relaxed a bit, as shown in 8], where Jambu and Papadima provide 
a version of the LCS formula for hypersolvable arrangements. An arrangement $\mathcal{A}$ is hypersolvable iff there is a supersolvable arrangement $\mathcal{B}$ and linear subspace $W$ with $\mathcal{A}=\mathcal{B} \cap W$ preserving the rank two flats (see [5]). Since an arrangement in $\mathbb{P}^{2}$ is supersolvable iff there exists $x \in \mathcal{L}_{2}(\mathcal{A})$ such that for each $y \in \mathcal{L}_{2}(\mathcal{A})$ there is a line of $\mathcal{A}$ connecting $x$ and $y$, it is clear that supersolvable arrangements have very constrained geometry.

The second generalization lies at the opposite end of the spectrum. Following Papadima and Suciu [13, define an arrangemnet to be decomposable if the holonomy Lie algebra behaves in degree 3 like a direct product of holonomy Lie algebras of pencil arrangements. Arrangements of this type are as simple as possible in many respects; as shown by Cohen-Suciu [3], the linearized Alexander invariant is a direct sum of submodules corresponding to Alexander invariants of rank two subarrangements, with scalars suitably extended. While the underlying arrangement $\mathcal{A}$ is not in general a product, the LCS ranks behave as if it were. Papadima-Suciu prove that for decomposable arrangements,

$$
U_{G}(t)=(1-t)^{b_{1}} \prod_{p \in L_{2}(\mathcal{A})} \frac{1-\mu(p) t}{1-t} .
$$

Here $\mu$ denotes the Möbius function: for $p \in L_{2}(\mathcal{A}), \mu(p)$ is one less than the number of hyperplanes of $\mathcal{A}$ which contain $p$.

Remark 1.5. Theorem 6.4 of [16] shows that a graphic arrangement has a cohomology ring which is Koszul iff the graph is supersolvable, and in [14, Papadima and Suciu give necessary and sufficient conditions for a graphic arrangement to be hypersolvable. Results of Stanley [19] and Terao [22] show that that $\mathcal{A}_{G}$ is supersolvable iff $G$ is chordal. As noted in Remark 6.18 of [16], the chromatic polynomial of a chordal graph is given by

$$
\chi_{\mathrm{G}}(t)=t^{\kappa_{0}} \prod_{j=1}^{\kappa_{0}-1}\left(1-j t^{-1}\right)^{\sum_{s=j}^{\kappa_{0}-1}(-1)^{s-j}\left(\begin{array}{l}
s \\
j
\end{array}\right) \kappa_{s}},
$$

and in this case, (1.2) specializes to the Falk-Randell LCS formula. At the opposite end of the spectrum are the decomposable graphic arrangements, classified in [13]: they are exactly those with $\kappa_{i}=0$ for all $i \geq 3$. For these arrangements, (1.2) specializes to (1.3).

The point of this paper is that for certain special classes of arrangements, it is possible to find a formula which encompasses both the decomposable and supersolvable classes, and indeed, everything in between. The main tool involved in the proof is a careful analysis of the holonomy Lie algebra, which we quickly review.

1.6. The holonomy Lie algebra and formal spaces. Let $X$ be the complement of a complex hypersurface in $\mathbb{P}^{n}$. Cup product gives a map $H^{1}(X, \mathbb{Q}) \wedge$ $H^{1}(X, \mathbb{Q}) \longrightarrow H^{2}(X, \mathbb{Q})$; the dual of this map is the comultiplication map

$$
H_{2}(X, \mathbb{Q}) \stackrel{\cup^{t}}{\longrightarrow} H_{1}(X, \mathbb{Q}) \wedge H_{1}(X, \mathbb{Q}) \longrightarrow \mathbb{L}\left(H_{1}(X, \mathbb{Q})\right),
$$

where $\mathbb{L}\left(H_{1}(X, \mathbb{Q})\right)$ is the free Lie algebra on $H_{1}(X, \mathbb{Q})$. Following Chen $[2$, define the holonomy Lie algebra of $X$ as the quotient $\mathbb{L}\left(H_{1}(X, \mathbb{Q})\right) / I_{X}$, where $I_{X}$ is the ideal generated by the image of the comultiplication map. In the case of arrangements, Kohno shows that the image of the comultiplication map is generated by all 
brackets of the form

$$
\left[x_{j}, \sum_{i=1}^{k} x_{i}\right],
$$

where $x_{i}$ is the generator of $\mathbb{L}\left(H_{1}(X, \mathbb{Q})\right)$ corresponding to the $i$-th hyperplane $e_{i}$ and $e_{j} \in\left\{e_{1}, \ldots, e_{k}\right\}$ is a (maximal) set of hyperplanes intersecting in codimension two; $\left\{e_{1}, \ldots, e_{k}\right\}$ corresponds to an element of $\mathcal{L}_{2}(\mathcal{A})$. This is also an immediate consequence of the description of the cohomology ring of an arrangement complement given by Orlik-Solomon in [12.

The proof of Kohno's main result runs as follows. In 21, Sullivan introduced the minimal model of a differential graded algebra (DGA) and associated notion of formality. A space $X$ is formal if the minimal model of the DGA of $\mathbb{Q}$-polynomial forms on $X$ is formal. Sullivan shows that for a formal space, $\left(\oplus G_{i} / G_{i+1}\right) \otimes \mathbb{Q}$ is isomorphic to the associated graded Lie algebra $\mathcal{L}$ of a filtration $\mathcal{L}_{i}$ arising in the construction of the 1-minimal model.

If $\mathcal{A}$ is defined by the vanishing of $\left\{\alpha_{1}, \ldots, \alpha_{n}\right\}$, let $\mathcal{R}=\mathbb{C}\left[\frac{d \alpha_{1}}{\alpha_{1}}, \ldots, \frac{d \alpha_{n}}{\alpha_{n}}\right]$. Brieskorn [1] shows that the map $\mathcal{R} \rightarrow H^{*}(X)$ sending $\frac{d \alpha_{i}}{\alpha_{i}}$ to its class is an isomorphism, so an arrangement complement $X$ is formal and Sullivan's results apply. Using work of [4] and [1], Kohno analyzes the bigrading on the Hirsch extensions appearing in the construction of the 1-minimal model and proves that there is an isomorphism from the holonomy Lie algebra to $\mathcal{L}$. It follows from Poincaré-BirkhoffWitt that that $\prod_{k=1}^{\infty}\left(1-t^{k}\right)^{-\phi_{k}}$ is the Hilbert series of the universal enveloping algebra of the holonomy Lie algebra.

\section{GLuing $\mathfrak{h}_{X}$ From SubgraphS}

In this section, we prove an amalgamation lemma, which will allow us to relate the holonomy Lie algebras of two graphic arrangements, identified along a common subgraph of a special type.

We denote by $I_{G}$ the ideal in $\mathbb{L}\left(H_{1}(X, \mathbb{Q})\right)$ describing the holonomy Lie algebra for the graphic arrangement $\mathcal{A}_{G}$. In this case, the brackets generating the ideal are as follows: either they have the form $\left[x_{1}, x_{2}\right]$ for edges $e_{1}, e_{2}$ not contained in a triangle of $G$, or they have the form $\left[x_{i}, x_{1}+x_{2}+x_{3}\right], i=1,2,3$, if $\left\{e_{1}, e_{2}, e_{3}\right\}$ is a triangle in $G$. Note that in the latter case it suffices to use the brackets $\left[x_{1}, x_{2}+x_{3}\right]$ and $\left[x_{2}, x_{1}+x_{3}\right]$.

To simplify notation, we write $\mathfrak{h}_{G}$ for $\mathfrak{h}_{\mathcal{A}_{G}}$ and $\mathbb{L}(G)$ for the free Lie algebra on the edges of $G$; edges of a graph will be denoted by $e_{j}$, with $x_{j}$ the corresponding generator in $\mathbb{L}(G)$ or $\mathfrak{h}_{G}$. Let $\Delta_{G}$ denote set of triangles in a graph $G$.

Definition 2.1. For a subgraph $K$ of $G$, the pair $(G, K)$ is triangle-complete (TC) if whenever $\left\{e_{i_{1}}, e_{i_{2}}, e_{i_{3}}\right\} \in \Delta_{G}$ and $\left\{e_{j_{1}}, e_{j_{2}}\right\} \subseteq K$ with $\left\{e_{j_{1}}, e_{j_{2}}\right\} \subseteq\left\{e_{i_{1}}, e_{i_{2}}, e_{i_{3}}\right\}$, then $\left\{e_{i_{1}}, e_{i_{2}}, e_{i_{3}}\right\} \subseteq K$. So if two edges of a triangle of $G$ lie in $K$, the third edge is also in $K$.

Lemma 2.2. Let $b=\left[x_{n}\left[x_{n-1},\left[x_{n-2},\left[\ldots\left[x_{1}, x_{0}\right]\right]\right] \ldots\right] \in \mathfrak{h}_{G}\right.$ be a bracket of depth $n$, and suppose that $e_{i} \in G \backslash K$ for some $i \in\{0, \ldots, n\}$. If $(G, K)$ is $T C$, then $b$ can be written as a sum of brackets of depth $n$

$$
b=\sum\left[x_{j_{n}},\left[x_{j_{n-1}},\left[x_{j_{n-2}},\left[\ldots\left[x_{j_{1}}, x_{j_{0}}\right]\right]\right] \ldots\right]\right.
$$

with all the $e_{j_{k}}$ 's in $G \backslash K$. 
Proof. We induct on the depth of the bracket. Consider $\left[x_{1}, x_{0}\right]$, with $x_{1} \in G \backslash K$ and $x_{0} \in K$. If $\left\{e_{1}, e_{0}\right\}$ is not the edge of a triangle in $G$, then $\left[x_{1}, x_{0}\right]=0$ in $\mathfrak{h}_{G}$, and we can replace $x_{0}$ with $x_{1}$. Otherwise, there exists an edge $e_{j}$ with $\left\{e_{1}, e_{0}, e_{j}\right\} \in \Delta_{G}$, so that in $\mathfrak{h}_{G}$ we have $\left[x_{1}, x_{0}+x_{j}\right]=0$, hence $\left[x_{1}, x_{0}\right]=\left[x_{j}, x_{1}\right]$. Since $(G, K)$ is TC, the assumption that $e_{1} \notin K$ implies that $e_{j} \notin K$.

Let $B$ denote a bracket of the form $\left[x_{n-1},\left[x_{n-2},\left[\ldots\left[x_{1}, x_{0}\right]\right]\right] \ldots\right]$, and consider $\left[x_{n}, B\right]$. If $B$ has some element in $G \backslash K$, then by induction we can expand $B$ as a sum of brackets of the desired type. So it suffices in this case to prove the result for $\left[x_{n},\left[x_{n-1}, B^{\prime}\right]\right]$ with all entries of $\left[x_{n-1}, B^{\prime}\right]$ in $G \backslash K$. But

$$
\left[x_{n},\left[x_{n-1}, B^{\prime}\right]\right]=-\left[B^{\prime},\left[x_{n}, x_{n-1}\right]\right]-\left[x_{n-1},\left[B^{\prime}, x_{n}\right]\right],
$$

and the result holds by induction. If $B$ is has no element in $G \backslash K$, then $x_{n} \in G \backslash K$, and again the result follows from the Jacobi identity (2.1) and induction.

Corollary 2.3. If $(G, K)$ is $T C$, then there are canonical surjections $\pi: \mathfrak{h}_{G} \rightarrow \mathfrak{h}_{K}$ and $I_{G} \rightarrow I_{K}$. Furthermore, the kernel $K(\pi)$ of $\pi$ is the subalgebra of $\mathfrak{h}_{G}$ generated by $\left\{x_{i} \mid e_{i} \in G \backslash K\right\}$.

Proof. The map $\mathrm{E}(G) \rightarrow \mathbb{L}(K)$, which is the identity on the edges of $K$ and sends all other edges to zero, extends to a surjection of free Lie algebras $\mathbb{L}(G) \stackrel{\psi}{\longrightarrow} \mathbb{L}(K)$. Since $K \subset G$, one can consider $\mathbb{L}(K)$ as a subalgebra of $\mathbb{L}(G)$, and $\psi$ restricts to the identity on $\mathbb{L}(K)$. The induced surjection $\mathbb{L}(G) \rightarrow \mathfrak{h}_{K}$ descends to a surjection $\pi: \mathfrak{h}_{G} \rightarrow \mathfrak{h}_{K}$, since the TC property and Lemma 2.2 guarantee that any generator of $I_{G}$ either is sent to zero, or is unchanged and hence is a generator of $I_{K}$. This not only gives the surjectivity of $\psi_{\mid I_{G}}: I_{G} \rightarrow I_{K}$, but also shows that

$$
I_{G} \cap \mathbb{L}(K)=I_{K}
$$

whenever $(G, K)$ is TC. Using the snake lemma, one concludes that $K(\pi)$ is the ideal in $\mathfrak{h}_{G}$ generated by $\left\langle x_{i} \mid e_{i} \in G \backslash K\right\rangle$. We now proceed to show it is indeed the subalgebra generated by these elements.

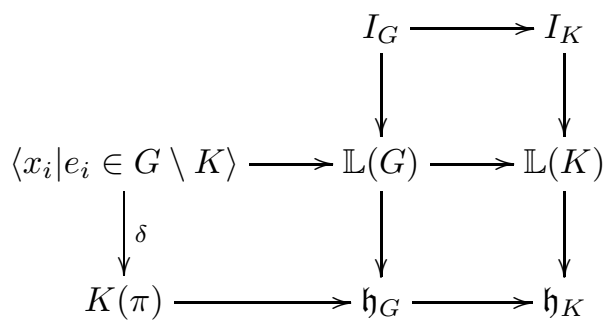

By Lemma 2.2 any bracket $\gamma \in K(\pi)$ involving some $x_{i}$ with $e_{i} \in G \backslash K$ may be written as a sum of brackets with all entries coming from $G \backslash K$, so it suffices to show that if $\alpha \neq 0 \in \mathfrak{h}_{G}$ is a sum of brackets involving only $x_{i}$ 's with $e_{i} \in K$ then $\alpha \notin K(\pi)$. Indeed, an element $\alpha$ of this form can be lifted to an element $\widehat{\alpha} \in \mathbb{L}(K) \subset \mathbb{L}(G)$, and hence $\pi(\alpha)=0$ if and only if $\widehat{\alpha} \in I_{K}=I_{G} \cap \mathbb{L}(K) \subset I_{G}$. This gives $\alpha=0$. 
Let $G_{1}$ and $G_{2}$ be two graphs, having a common subgraph $K$. We construct a new graph $G=G_{1} \bigcup_{K} G_{2}$ by identifying $G_{1}$ and $G_{2}$ along the image of $K$.

Theorem 2.4. If $G=G_{1} \bigcup_{K} G_{2}$ and $\left(G_{i}, K\right)$ and $\left(G, G_{i}\right)$ have property $T C$ for $i \in\{1,2\}$, then:

a: The diagram

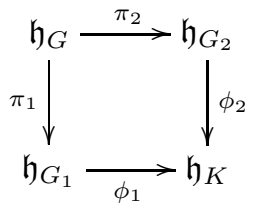

is a pull-back diagram of Lie algebras.

b: The diagram

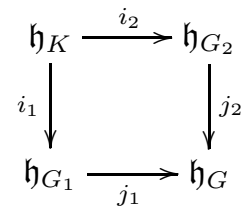

is a push-out diagram of Lie algebras.

Proof. To prove assertion a, it suffices to show that

$$
0 \longrightarrow \mathfrak{h}_{G} \stackrel{\pi_{1} \times \pi_{2}}{\longrightarrow} \mathfrak{h}_{G_{1}} \times \mathfrak{h}_{G_{2}} \stackrel{\phi_{1}-\phi_{2}}{\longrightarrow} \mathfrak{h}_{K} \longrightarrow 0 .
$$

is an exact sequence of vector spaces. It follows from Corollary 2.3 that $\phi_{1}-\phi_{2}$ is surjective. On the other hand, Lemma 2.2 shows that any element $\alpha_{i} \in \mathfrak{h}_{G_{i}}$, $i=1,2$, can be written as $\alpha_{i}=g_{i}+k_{i}$, where $g_{i}$ (respec. $k_{i}$ ) is a sum of brackets involving only generators associated to edges in $G_{i}-K$ (respec. $K$ ). In particular, if $\left(\alpha_{1}, \alpha_{2}\right) \in \operatorname{Ker}\left(\phi_{1}-\phi_{2}\right)$ then $k_{1}=k_{2}$ and one immediately sees that $\left(\alpha_{1}, \alpha_{2}\right)$ is in the image of $\pi_{1} \times \pi_{2}$. The exactness of the sequence in the middle is now clear. Consider the commutative diagram:

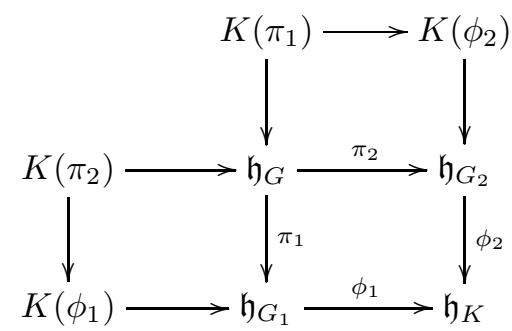

Since both $\left(G_{i}, K\right)$ and $\left(G, G_{i}\right)$ are TC, it follows from Lemma 2.2 that any bracket in $K\left(\phi_{2}\right)$ may be written as a bracket involving only the edges in $G_{2} \backslash K=$ $G \backslash G_{1}$, and any bracket in $K\left(\phi_{1}\right)$ may be written as a bracket involving only the edges in $G_{1} \backslash K=G \backslash G_{2}$. Thus, the maps $K\left(\pi_{1}\right) \rightarrow K\left(\phi_{2}\right)$ and $K\left(\pi_{2}\right) \rightarrow K\left(\phi_{1}\right)$ are surjective.

By Corollary 2.3 $K\left(\pi_{i}\right)$ is the subalgebra of $\mathfrak{h}_{G}$ generated by the variables associated to edges in $G \backslash G_{i}, i=1,2$ and, similarly, $K\left(\phi_{i}\right)$ is the subalgebra of $\mathfrak{h}_{G}$ generated by the variables associated to edges in $G_{i} \backslash K, i=1,2$. 
It follows that the projection $\mathbb{L}(G) \rightarrow \mathfrak{h}_{G}$ induces an isomorphism

$$
\mathbb{L}\left(G \backslash G_{1}\right) / I_{G} \cap \mathbb{L}\left(G \backslash G_{1}\right) \simeq K\left(\pi_{1}\right) .
$$

The surjection $\mathbb{L}(G) \rightarrow \mathbb{L}\left(G_{2}\right)$ restricts to an isomorphism $\mathbb{L}\left(G \backslash G_{1}\right) \stackrel{\simeq}{\longrightarrow} \mathbb{L}\left(G_{2} \backslash K\right)$ and, under this isomorphism one has

$$
\begin{aligned}
K\left(\pi_{1}\right) & \simeq \mathbb{L}\left(G \backslash G_{1}\right) / I_{G} \cap \mathbb{L}\left(G \backslash G_{1}\right) \simeq \mathbb{L}\left(G_{2} \backslash K\right) / I_{G} \cap \mathbb{L}\left(G_{2}\right) \cap \mathbb{L}\left(G_{2} \backslash K\right) \\
& =\mathbb{L}\left(G_{2} \backslash K\right) / I_{G_{2}} \cap \mathbb{L}\left(G_{2} \backslash K\right) \simeq K\left(\phi_{2}\right),
\end{aligned}
$$

where the equality comes from (2.2), since $\left(G, G_{2}\right)$ is TC. It follows that the upper horizontal arrow in the diagram is an isomorphism, and so is the leftmost vertical arrow. In particular $K\left(\pi_{1}\right) \cap K\left(\pi_{2}\right)=0$, thus showing that the kernel of $\pi_{1} \times \pi_{2}$ is trivial, and this concludes the proof that the sequence (2.3) is exact, and (a) follows.

To prove assertion $\mathbf{b}$, first observe that (2.2) implies that the natural maps $i_{r}: \mathfrak{h}_{K} \rightarrow \mathfrak{h}_{G_{r}}$ and $j_{r}: \mathfrak{h}_{G_{r}} \rightarrow \mathfrak{h}_{G}$ are injections, for $r=1,2$. Now, consider the sequence

$$
0 \longrightarrow \mathfrak{h}_{K} \stackrel{i_{1} \times i_{2}}{\longrightarrow} \mathfrak{h}_{G_{1}} \times \mathfrak{h}_{G_{2}} \stackrel{j_{1}-j_{2}}{\longrightarrow} \mathfrak{h}_{G} \longrightarrow 0 .
$$

A similar argument to the one used in previous assertion shows that this sequence is exact and that the diagram is in fact a push-out of Lie algebras.

Corollary 2.5. With assumptions above,

$$
U_{G}(t)=\frac{U_{G_{1}}(t) \cdot U_{G_{2}}(t)}{U_{K}(t)} .
$$

\section{MAIN TheOREM}

To prove the main theorem, we induct on the number of vertices $v(G)$ of $G$, the basic case being trivial. If $G$ is a graph on $\left\{v_{1}, \ldots, v_{n}\right\}$, then let $G_{1}$ denote the subgraph of $G$ consisting of all edges of $G$ not containing $v_{n}$, and $G_{2}^{\prime}$ denote the subgraph of $G$ consisting of all edges of $G$ containing $v_{n}$. Note that $v\left(G_{1}\right)=n-1$.

Lemma 3.1. Let $K$ consist of all edges in $G_{1}$ which connect vertices $\left\{a_{1}, a_{2}\right\}$ such that $\left\{a_{1}, v_{n}\right\},\left\{a_{2}, v_{n}\right\} \in G_{2}^{\prime}$, and define $G_{2}=G_{2}^{\prime} \cup K$. Then $\left(G_{i}, K\right)$ and $\left(G, G_{i}\right)$ are $T C$.

Proof. If a triangle with vertices $\left\{a_{1}, a_{2}, a_{3}\right\}$ of $G$ has two edges $\left\{a_{1}, a_{2}\right\},\left\{a_{1}, a_{3}\right\}$ in $K$, then $\left\{a_{i}, v_{n}\right\} \in G_{2}^{\prime}$ and hence $\left\{a_{2}, a_{3}\right\} \in K$. So $(G, K)$ is TC, which implies $\left(G_{i}, K\right)$ is TC. Clearly $\left(G, G_{1}\right)$ is TC, since any triangle of $G$ with two edges in $G_{1}$ does not contain the vertex $v_{n}$, so has the third edge in $G_{1}$. Finally, if a triangle of $G$ has two edges $\left\{a_{1}, a_{2}\right\},\left\{a_{1}, a_{3}\right\}$ in $G_{2}$, then there are three possibilities.

(1) If $v_{n}=a_{1}$, then $\left\{a_{2}, a_{3}\right\} \in K \subseteq G_{2}$.

(2) If $v_{n}=a_{2}$, then $\left\{a_{1}, a_{3}\right\} \in K$, hence $\left\{v_{n}, a_{3}\right\} \in G_{2}$.

(3) If $v_{n} \notin\left\{a_{1}, a_{2}, a_{3}\right\}$, then $\left\{v_{n}, a_{2}\right\},\left\{v_{n}, a_{3}\right\} \in G_{2}$, so $\left\{a_{2}, a_{3}\right\} \in K \subseteq G_{2}$. 
If $G \simeq K_{n}$ then the formula holds by [10], so we may assume $G \not K_{n}$. Using the notation of Lemma 3.1 choose $v_{n}$ so that $v\left(G_{2}\right)=v\left(G_{2}^{\prime}\right)<v(G)$. By induction, Lemma 3.1 and Corollary 2.5 we find that $U_{G}(t)=$

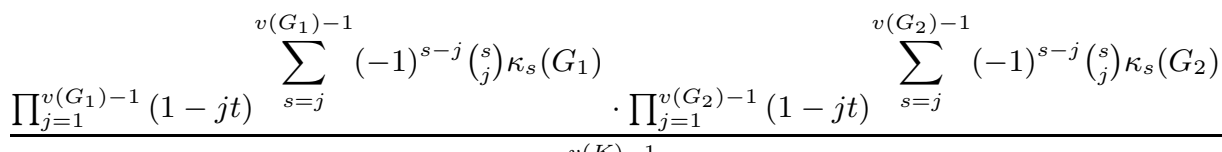

$$
\begin{aligned}
& \prod_{j=1}^{v(K)-1}(1-j t) \sum_{s=j}^{v(K)-1}(-1)^{s-j}\left(\begin{array}{l}
s \\
j
\end{array}\right) \kappa_{s}(K)
\end{aligned}
$$

Observing that for any graph $H$, one has $\kappa_{s}(H)=0$ whenever $s \geq v(H)$, we can write this formula as

$$
\prod_{j \geq 1}(1-j t)\left[\sum_{s \geq j}(-1)^{s-j}\left(\begin{array}{l}
s \\
j
\end{array}\right)\left\{\kappa_{s}\left(G_{1}\right)+\kappa_{s}\left(G_{2}\right)-\kappa_{s}(K)\right\}\right] .
$$

Partition the complete subgraphs of $G$ into two sets: those which contain $v_{n}$, and those which do not. A complete subgraph lying in $G_{2}$ and not containing $v_{n}$ corresponds to a complete subgraph lying in $K$, and so is counted in both $G_{1}$ and $G_{2}$. This concludes the proof of (1.2). Notice that the base case (1.1) can be proved directly, using the results of [6], [19] and [22].

Remark 3.2. The ideas here may be extended to the class of Lie algebras where an analog of Theorem 2.4 holds, and will appear elsewhere shortly.

\section{REFERENCES}

[1] E. Brieskorn, Sur les groupes de tresses, Séminaire Bourbaki, 1971/72, LNM 317, SpringerVerlag, (1973), 21-44.

[2] K.T. Chen, Iterated integrals of differential forms and loop space cohomology, Ann. of Math. 97 (1973), 217-246.

[3] D. Cohen, A. Suciu, Alexander invariants of complex hyperplane arrangements, Trans. Amer. Math. Soc. 351 (1999), 4043-4067.

[4] P. Deligne, Theorie de Hodge II, Inst. Hautes Etudes Sci. Publ. Math. 40 (1971), 5-58.

[5] G. Denham, A. Suciu, On the homotopy Lie algebra of an arrangement, Michigan Math. J. 54 (2006), 319-340.

[6] M. Falk, R. Randell, The lower central series of a fiber-type arrangement, Invent. Math. 82 (1985), 77-88.

[7] H. Hamm, D. T. Lê, Un théorème de Zariski du type de Lefschetz, Ann. Sci. École Norm. Sup. 6 (1973), 317-366.

[8] M. Jambu, S. Papadima, A generalization of fiber-type arrangements and a new deformation method, Topology 37 (1998), 1135-1164.

[9] T. Kohno, On the holonomy Lie algebra and the nilpotent completion of the fundamental group of the complement of hypersurfaces, Nagoya Math J. 92 (1983), 21-37.

[10] T. Kohno, Série de Poincaré-Koszul associée aux groupes de tresses pures, Invent. Math. 82 (1985), 57-75.

[11] J. Morgan, The algebraic topology on smooth algebraic varieties, Publ. Math. I.H.E.S. 48 (1978), 137-204.

[12] P. Orlik, L. Solomon, Combinatorics and topology of complements of hyperplanes, Invent. Math. 56 (1980), 167-189.

[13] S. Papadima, A. Suciu, When does the associated graded Lie algebra of an arrangement group decompose?, Commentarii Mathematici Helvetici, 81 (2006), 859-875. 
[14] S. Papadima, A. Suciu, Higher homotopy groups of complements of complex hyperplane arrangements Adv. Math. 165 (2002), 71-100.

[15] S. Papadima, S. Yuzvinsky, On rational $K[\pi, 1]$ spaces and Koszul algebras, J. Pure Applied Algebra 144 (1999), 157-167.

[16] H. Schenck, A. Suciu, Lower central series and free resolutions of hyperplane arrangements, Trans. Amer. Math. Soc. 354 (2002), 3409-3433.

[17] H. Schenck, A. Suciu, Resonance, linear syzygies, Chen groups, and the Bernstein-GelfandGelfand correspondence, Trans. Amer. Math. Soc., 358, (2006), 2269-2289.

[18] B. Shelton, S. Yuzvinsky, Koszul algebras from graphs and hyperplane arrangements, J. London Math. Soc. 56 (1997), 477-490.

[19] R.P. Stanley, Supersolvable lattices, Algebra Universalis 2 (1972), 197-217.

[20] A. Suciu, Fundamental groups of line arrangements: Enumerative aspects, in: Advances in algebraic geometry motivated by physics, Contemporary Math., vol. 276, Amer. Math. Soc, Providence, RI, 2001, pp. 43-79.

[21] D. Sullivan, Infinitesimal computations in Topology, Publ. Math. I.H.E.S. 47 (1977), 269-331.

[22] H. Terao, Modular elements of lattices and topological fibration, Adv. Math. 62 (1986), 135154.

Department of Mathematics, Texas A\&M University, College Station, TX 77843

E-mail address: plfilho@math.tamu.edu

$U R L:$ http://www.math.tamu.edu/ paulo.lima-filho

Department of Mathematics, University of Illinois, Urbana, IL 61801

E-mail address: schenck@math.uiuc.edu

$U R L:$ http://www.math.uiuc.edu/ schenck 